\title{
Design of Bicman Speed Based on C51 Microcontroller
}

\author{
Yang Zhan \\ Jilin Agricultural University, Changchun, China
}

\begin{abstract}
This paper designed and produced a device based on c51 MCU. This device recorded the number of cycles of a Bicman tire in a certain time though the Hall sensor, and then calculated the mileage and speed of the Bicman by the number of cycles. Finally it could display the mileage and speed with a digital tube. It also has a speeding alert function. The device can be applied to the instrument of the electric bicycle, motorcycle, automobile and other motor vehicles.
\end{abstract}

Keywords-MCU; hall sensor; speed

\section{INTRODUCTION}

The speed measurement can be used to measure the pulse width and the method of counting. The so-called counting method is given a gate-time. Number of input pulses at gate time, the pulse width of the signal to be measured is controlled by the pulse width of the signal to be measured, Counting of a high precision high frequency signal. Because the gate and the measured signal can't be synchronized, therefore, this method was problematic \pm 1 error. The counting method is suitable for high frequency signal measurement. Pulse width measurement is suitable for low frequency signal measurement.

Use microcontroller to measure the speed of a Bicman, you can use simple pulse counting method, as long as each revolution of the shaft, resulting in a fixed or a plurality of pulses and pulse into the microcontroller to count, you can get speed information. Hall sensors are sensitive to the magnetic sensor element, commonly used in the switching signal acquisition, such as the CS3020 and the like. The sensor circuit using CS3020, which is a magnetic sensor circuit consists of a voltage regulator, Hall voltage generator, differential amplifier, Schmitt trigger and open collector output stage, the input for the magnetic flux density, the output It is a digital voltage signal.

This sensor is a three-terminal device, similar to the shape of the transistor, just plug in the power, ground, can work, wide operating voltage range, easy to use. Hall sensor obtained using a pulse signal, the mechanical structure can be made simple, as long as the glue a magnet on the circumference of the shaft, so that the Hall switch near the magnet, there is signal output shaft is rotated, it will constantly generates a pulse signal output. This sensor is a solid structure, small size, light weight, long life, easy installation, low power consumption, high frequency (up to 1MHZ), shock-resistant, not afraid of contaminated dust, oil, and moisture and salt spray corrosion or the like.

\section{DESIGN OF SYSTEM HARDWARE CIRCUIT}

Bicman speed tester using C51 microcontrollers for controller ${ }^{[1]}$, the mileage / speed sensor using Hall components CS3020, hardware circuit diagram shows in figure I.

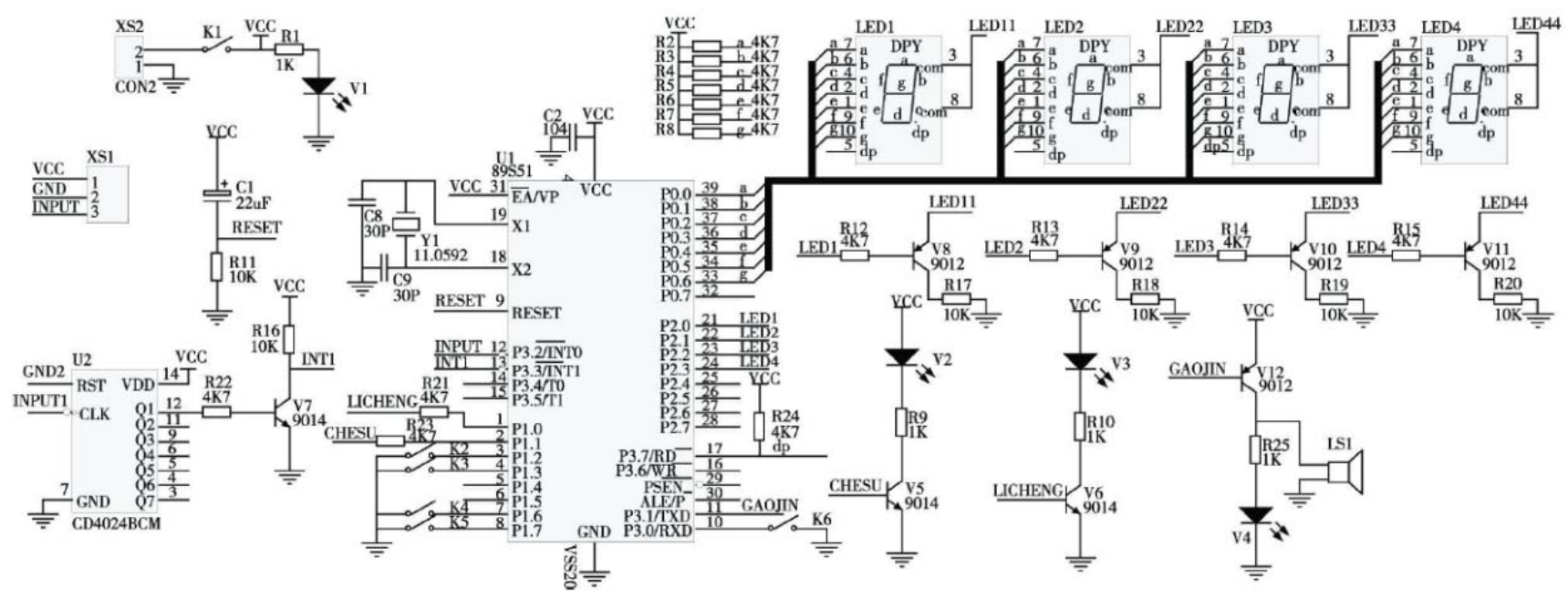

FIGURE I. THE CIRCUIT DIAGRAM OF HARDWARE 
In C51 microcontroller, P0 port for the seven-segment LED display (LED1- LED4) segment code control signal, P2, for seven-segment LED display (LED1 - LED4) bit code control signals. When displaying mileage, third decimal placed by the P3.7 port control lights. When the LED of V3 P1.0 port lights up, seven-segment LED display indicates the mileage. When the LED of V2 P1.1 port lights up, seven-segment LED display indicates the vehicle speed. P1.2, P1.3, P1.6 and P1.7 are used to set the size of the rim. P3.0 port switch K6 is used to determine the display function: When the switch K6 is closed, seven-segment LED display shows speed; when the switch K6 is open, seven-segment LED display shows the mileage. External interrupt INT0 for counting the number of turns of the wheel, when the wheel magnet $S$ pole very close to the probe when the Hall sensor, Hall sensors on output a low pulse, MCU enters INT0 external interrupt service program ${ }^{[2]}$, count the number of wheel rotation cycles. The interrupt INT1 of thirteenth foot for controlling start and stop timer T1, when the input is 0:00 off timer, the control signal is a pulse counting wheel rotation number of laps after CD4024 divided by two to produce the divider, Thus, each time the timer start and stop time $\mathrm{T} 1$ is just one turn of the wheel of time, according to the circumference of the wheel can be calculated Bicman speed. Eleventh foot for output speeding warning signal [3], when speeding, buzzer chirping and LS1 LED V4 is lit. XS1 access socket for Hall sensor, XS2 for system power supply input, it is $+5 \mathrm{~V}, \mathrm{LED} \mathrm{V} 1$ is the power indicator.

\section{THE MAIN SYSTEM PROGRAM}

The main program flow sheet shows in Figure II. First, start the initialization process, and then detect the level of SCM P3.0 port, if P3.0 = 1, then calling the subprogram of display speed, speed calculation results were displayed on the digital pipe; if P3.0 $=0$, then calling the subprogram of display mileage, mileage calculation results were displayed on digital tube.

\section{A. Initialization Program}

It mainly completes the following work: set the T1 to the external control timer mode; set the external interrupt INT0 and INT1 to edge triggered mode ${ }^{[4]}$; cleared part of the memory unit; set the wheel circumference value; opened timer and interrupt.

\section{B. The Program for the Wheel Error}

When the error of the wheel occurred, it could output a cycle of $0.5 \mathrm{~S}$ square wave signal from the P3.1 port, the LED V4 was lighted and flashed. At the same time, buzzer LS1 sounded to remind.

\section{The Main Program}

In the main program, according the state of P3.0 port to chosen the seven segment LED display mileage or speed.

\section{Program for Calculating Mileage}

It counted the number of turns of the wheel, which was the hex counter. The $60 \mathrm{H}$ was low, the $62 \mathrm{H}$ was high.

\section{E. The Service Program for INT0 and INT1}

It was used to processing the wheel to rotate a circle after the timing data: when the flag bit (00h) was 1 , indicating that the counter overflow, then put the maximum time value
(\#0FFH); when the flag (00h) bit was 0 , put the values of count unit (TL1 and Th1, 6CH, 6DH) into 68H, 69H, 6ah, 6bh unit. The following codes are the program of INT0 and INT1.

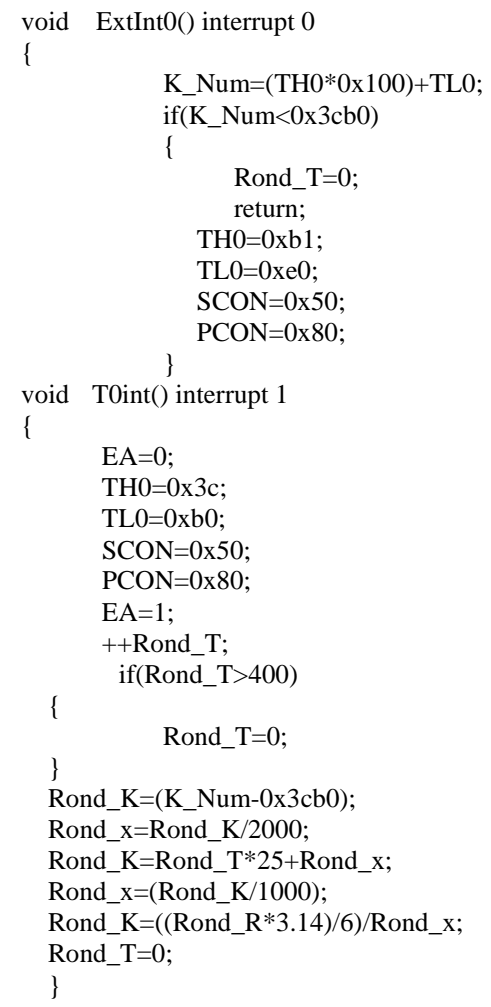

\section{F. Display Subprogram}

When the mileage was displayed, first calculated the number of laps counter data, calculate the total mileage. When the speed was displayed, calculated the value of the circumference of the wheel was divided by the time of rotation, and then converted into km / hour. Finally put in from $70 \mathrm{H}$ to $73 \mathrm{H}$ unit for data display.

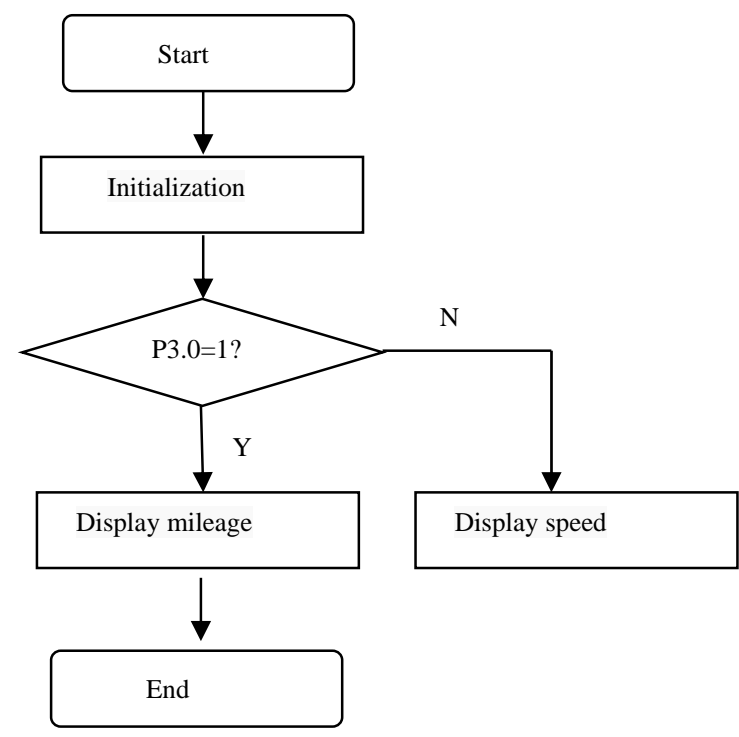

FIGURE II. THE MAIN SYSTEM PROGRAM FLOW SHEET 


\section{INSTALLATION INSTRUCTIONS}

The magnet was fixed on the steel wire of the front wheel, fixed the probe Hall sensor on the proper position of a Bicman fork, to ensure that each revolution of the front whee ${ }^{[5]}$, the magnet wire on the $\mathrm{S}$ pole and the Hall sensor on the Bicman fork probe device to meet once.

\section{CONCLUSION}

Through the design of the hardware and software debugged, the technical parameters of the bicycle mileage / speed meter were meet the design requirements, the continuous operation of the software did not appear to crash and other abnormal phenomena. It is technically feasible. In addition, in order to make the mileage / speed meter function more perfect, it can be improved from the following aspects:

If we want to accumulate the mileage value after the power outage, we can use without loss of EEPROM data memory, such as the $\mathrm{I}^{2} \mathrm{C}$ bus interface with the 24 series EEPROM to achieve. Since there is no $\mathrm{I}^{2} \mathrm{C}$ C51 bus interface, then we can use two $\mathrm{I} / \mathrm{O}$ port lines to simulate the $\mathrm{I}^{2} \mathrm{C}$ bus interface.

To record the value of the Bicman mileage of more than 10 million meters, it can be widened digit seven-segment LED display to seven, which is the use of the MCU existing RAM data memory is very easy to implement.

To save energy as much as possible to extend battery life, we can added a touch of a button click, the system enters a power saving mode (seven-segment LED display does not light, mileage accumulated as usual), then click, and enter the normal operating mode. When the system is in normal display, the input current is approximately $50 \mathrm{~mA}$, when speeding buzzer LS1 calls, the input current is about $100 \mathrm{~mA}$. In power-saving mode, the input current is approximately $10 \mathrm{~mA}$. If we use the LCD display, it can greatly reduce system power consumption, but we must pay attention to the display brightness to ensure that bright light during the day can also be displayed clearly.

\section{REFERENCES}

[1] Liquan Han and Hua Yu, principle and application of single chip microcomputer, Beijing Hope Electronic Publishing House, Beijing: 2006, pp.2-3.

[2] Suohong Wang," Implementation of electronic speedometer Microcomputer” Foreign electronic components.2004(5), pp.7-8.

[3] Xiangheng Tang," Electronic vehicle odometer" Automobile electric appliance.2004(1),pp.10-11.

[4] Kan Liu and Yongtai Zhang,” ARM programming optimization strategy and technology" Application of single chip microcomputer and embedded system.2004(4),pp.23-26.

[5] Qihua Zhang,” TEM sample preparation technology suitable for deep trench structure observation” Semiconductor technology.2010 (2),pp.169-171. 